\title{
Cold collisions catalyse conformational conversion $\nmid$
}

\section{Undine Erlekam,* Marcin Frankowski, Gert von Helden and Gerard Meijer}

\author{
Received 8th March 2007, Accepted 15th May 2007 \\ First published as an Advance Article on the web 8th June 2007 \\ DOI: $10.1039 / b 703571 c$
}

\begin{abstract}
We have determined the abundance of two different conformational structures of the mixed benzene dimer $\left(\mathrm{C}_{6} \mathrm{H}_{6}\right)\left(\mathrm{C}_{6} \mathrm{D}_{6}\right)$ in a molecular beam, with various carrier gases. These two T-shaped conformers have a subtle zero-point energy difference of only a few $\mathrm{cm}^{-1}$, and a transition state barrier of about $64 \mathrm{~cm}^{-1}$. Nevertheless, depending on the carrier gas, the lowest energy conformer can exclusively be prepared in the molecular beam. Low-energy two-body collisions of the benzene-dimers with the carrier gas atoms are concluded to be responsible for this.
\end{abstract}

In order to investigate the intrinsic properties of molecules or clusters, it is desirable to study them at low temperatures and isolated from external perturbations. Molecular beam methods are frequently used for this. ${ }^{1}$ Many molecules or molecular complexes can adopt various conformational structures that differ in potential energy. In molecular beam experiments these different conformations are often found to co-exist. ${ }^{2,3}$ In particular, structures can be observed that have high potential energies and the observed structural distributions are therefore frequently not in thermal equilibrium with the other degrees of freedom. ${ }^{2-10}$ This can be rationalized by the height of the barriers that separate potential energy minima which might be hard to overcome under the conditions prevailing in the molecular beam experiments. The study of the potential energy landscape of gas-phase molecules and the development of experimental methods to manipulate the conformational distribution of these species currently is an active field of research. ${ }^{11,12}$

There has been a variety of studies on the dependence of the conformational distribution in a molecular beam on the experimental parameters. The question how the composition of the carrier gas influences the relative population of different conformers in a supersonic expansion has been addressed by Ruoff et al. in $1990 .^{4}$ In that work, microwave absorption measurements were performed $\left(T_{\text {rot }} \approx 3 \mathrm{~K}\right.$ ) to deduce relative populations of the possible conformational structures of a variety of molecules in molecular beams. In such measurements, the effect of changing the rotational temperature and the effect of changing the relative abundance of a specific conformer can appear the same, and it is non-trivial to distinguish between these effects. Nevertheless, one of the important observations of that work was that conversion between conformers, separated by barriers $\leq 350 \mathrm{~cm}^{-1}$, appeared to involve relatively long-range polarization effects and to take place relatively late in the expansion region. In a more recent study, Suhm and co-workers investigated the isomerism of jet-cooled, isotopically mixed methanol dimers

Fritz-Haber-Institut der Max-Planck-Gesellschaft, Faradayweg 4-6, D-14195 Berlin, Germany. E-mail: erlekam@fhi-berlin.mpg.de $\dagger$ The HTML version of this article has been enhanced with colour images.
$\left(\mathrm{CH}_{3} \mathrm{OH}-\mathrm{CH}_{3} \mathrm{OD}\right)$ in a supersonic jet expansion. ${ }^{10}$ They used Fourier transform infrared (FT-IR) absorption spectroscopy in the 3-4 $\mu \mathrm{m}(\mathrm{O}-\mathrm{H}$ and $\mathrm{O}-\mathrm{D}$ stretch) region to study the degree of conformer conversion (or: donor/acceptor isomerism) in their $10-20 \mathrm{~K}$ jet expansion. A complication in the latter studies is that it is difficult to spectroscopically distinguish the mixed dimers from their homodimer counterparts. For the mixed methanol dimer system spectra were only reported in a pure He expansion, and it was concluded that relaxation to the lowest energy conformer was incomplete in this case. In a recent theoretical study, the conformational changes of glycine in collisions with rare-gas atoms, with collision energies ranging from 100 to $1000 \mathrm{~K}$, were investigated. ${ }^{13}$ In that work it was shown that attractive interactions between the colliding atoms and the glycine molecule can lower the barrier between conformers, i.e. that these interactions can catalyse conformer conversion. A molecular dynamics simulation addressing the problem of thermodynamic versus kinetic control of isomers of dihalogen-rare gas complexes in a supersonic expansion has been presented by Bastida et al. ${ }^{14}$

In spite of all these studies, the microscopic mechanisms responsible for the conformational distributions are often not clear. We here present a study on the distribution of the conformers of the isotopically mixed benzene dimer $\left(\mathrm{C}_{6} \mathrm{H}_{6}\right)\left(\mathrm{C}_{6} \mathrm{D}_{6}\right)$ in a molecular beam at rotational temperatures of 1-2 K, using different carrier gases. A variety of theoreti$\mathrm{cal}^{15-20}$ and experimental ${ }^{21-29}$ studies exist, discussing benzene dimer structures with two symmetrically inequivalent subunits (T-shaped) and the possibility of a parallel displaced, a V-shaped or a sandwich structure with two symmetrically equivalent sites. However, Raman, ${ }^{26}$ microwave ${ }^{28}$ and recent infrared $^{29}$ experiments strongly support a T-shaped structure for this loosely bound Van der Waals complex. In that dimer, the two monomer units are inequivalent and do not interchange on the experimental timescale; one of the monomer units is in the "top" position whereas the other one is in the "stem". When one of the two rings is isotopically labeled, two isomers exist which can have a slightly different zero-point energy and which are separated by a barrier that is calculated to be $64 \mathrm{~cm}^{-1}$. ${ }^{19}$ As such, this is a very subtle system for the study of conformational conversion. Another important 
feature of this system is that one can unambiguously distinguish between the isomers with either the $\mathrm{C}_{6} \mathrm{H}_{6}$ unit or the $\mathrm{C}_{6} \mathrm{D}_{6}$ unit in the "stem" position, and that one can quantitatively determine the relative abundance of these isomers, via electronic spectroscopy. ${ }^{23}$ Moreover, by using mass-selective detection after $\left(1+1^{\prime}\right)$-resonance enhanced multi photon ionization (REMPI), any interference by the signal of the homodimer is avoided. In this system a pronounced influence of the carrier gas on the conformational distribution is observed. This can only be explained by a mechanism that involves low-energy collisions between the dimer and the carrier gas atoms, similar to the one described for the interconversion of isomers of dihalogen-rare gas complexes. ${ }^{14}$

Benzene dimer complexes are generated in a supersonic expansion of benzene $(0.03 \%)$ and helium or neon as a carrier gas at a stagnation pressure of two bars into vacuum. The molecular beam is skimmed and interrogated by two counterpropagating UV beams. Tuneable UV light is generated with two frequency doubled Nd:YAG pumped pulsed dye lasers. The ions generated in a two-colour-REMPI process are detected perpendicular to the molecular beam axis in a time of flight (TOF) mass spectrometer. For electronic excitation in the REMPI process, the $0_{0}^{0}$ transition to the first electronically excited state $S_{1}$ is used. The $0_{0}^{0}$ transition is symmetry forbidden in the benzene monomer, however, due to the broken symmetry weakly allowed in the benzene dimer. A second dye laser provides fixed frequency light around $35460 \mathrm{~cm}^{-1}$ used for ionization. The experiments are performed using a $50: 50$ mixture of $\mathrm{C}_{6} \mathrm{H}_{6}$ and $\mathrm{C}_{6} \mathrm{D}_{6}$. Therefore, dimers of three different masses can be generated: the two homodimers $\left(\mathrm{C}_{6} \mathrm{H}_{6}\right)_{2}$ and $\left(\mathrm{C}_{6} \mathrm{D}_{6}\right)_{2}$ and the heterodimer $\left(\mathrm{C}_{6} \mathrm{H}_{6}\right)\left(\mathrm{C}_{6} \mathrm{D}_{6}\right)$.

In Fig. 1 the UV spectra for the different benzene dimer species are shown in the region of the origin of the $\mathrm{S}_{1} \leftarrow \mathrm{S}_{0}$ transition using either helium (left) or neon (right) as a carrier gas. When monitoring $\left(\mathrm{C}_{6} \mathrm{H}_{6}\right)_{2}$ with helium as a carrier gas, a peak around $38042 \mathrm{~cm}^{-1}$ with a partially resolved splitting of about $2 \mathrm{~cm}^{-1}$ is observed (see Fig. 1(c)). For $\left(\mathrm{C}_{6} \mathrm{D}_{6}\right)_{2}$ a similar spectral feature is observed, shifted about $200 \mathrm{~cm}^{-1}$ to the blue (see Fig. 1(a)). When performing the experiments using helium as a carrier gas and monitoring the mass of the isotopically mixed benzene dimer, two single peaks occur, slightly shifted to the blue compared to the corresponding homodimer transitions (see Fig. 1(b)). A similar UV spectrum of the isotopically mixed benzene dimer has been measured several years ago by Bernstein and co-workers. ${ }^{24}$ In the T-shaped complex, the chromophore in the "top" is considerably more perturbed relative to the free benzene chromophore than the one in the "stem". In REMPI experiments, therefore, a narrow spectral feature is only observed when the excitation occurs on the "stem" molecule. ${ }^{26}$ Since the isotopic composition of the "stem" is different for the two isomers of the mixed benzene dimer, they can be selectively excited by choosing the appropriate UV frequency and thus detected separately. Ions generated using $38044 \mathrm{~cm}^{-1}$ light thus originate from dimers with a perprotonated "stem", indicated from now on as $\left(\mathrm{C}_{6} \mathrm{H}_{6}\right)^{\mathrm{S}}\left(\mathrm{C}_{6} \mathrm{D}_{6}\right)^{\mathrm{T}}$, whereas ions generated using light at 38246 $\mathrm{cm}^{-1}$ correspond to the isomer in which the "stem" is perdeuterated, $\left(\mathrm{C}_{6} \mathrm{D}_{6}\right)^{\mathrm{S}}\left(\mathrm{C}_{6} \mathrm{H}_{6}\right)^{\mathrm{T}}$. In helium, both isomers occur with similar intensities in the spectrum. Using neon as an

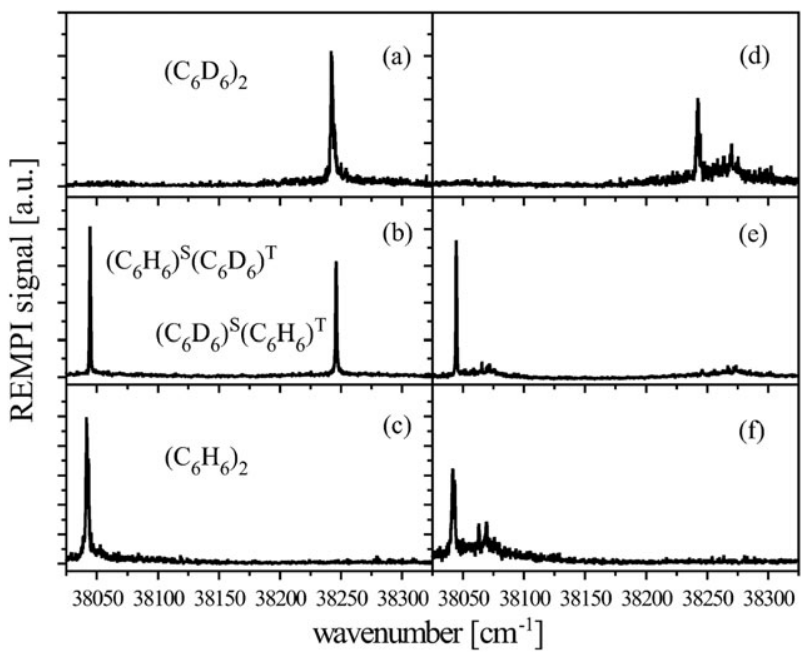

Fig. 1 Two-colour $\left(1+1^{\prime}\right)$-REMPI spectra of the benzene dimer around the origin of the $S_{1} \leftarrow S_{0}$ transition. The measurements are performed in a molecular beam using helium (left) and neon (right) as a carrier gas. The upper spectra are recorded on the mass of the perdeuterated homodimer, the middle spectra on the mass of the mixed dimer and the lower spectra on the mass of the perprotonated homodimer. When neon is used as a carrier gas, the mixed dimer with the perprotonated monomer unit in the "stem", the lowest energy $\left(\mathrm{C}_{6} \mathrm{H}_{6}\right)^{\mathrm{S}}\left(\mathrm{C}_{6} \mathrm{D}_{6}\right)^{\mathrm{T}}$ isomer, is exclusively observed.

expansion gas, and monitoring $\left(\mathrm{C}_{6} \mathrm{D}_{6}\right)_{2}$ or $\left(\mathrm{C}_{6} \mathrm{H}_{6}\right)_{2}$, again two slightly split peaks (Fig. 1(d) and (f)) at the same spectral positions as in the case of helium are observed. However, in contrast to the measurements in helium, for $\left(\mathrm{C}_{6} \mathrm{H}_{6}\right)\left(\mathrm{C}_{6} \mathrm{D}_{6}\right)$, only one peak is observed (see Fig. 1(e)). This peak corresponds to the $\left(\mathrm{C}_{6} \mathrm{H}_{6}\right)^{\mathrm{S}}\left(\mathrm{C}_{6} \mathrm{D}_{6}\right)^{\mathrm{T}}$ isomer and it appears that the other isomer with the "stem" deuterated and the "top" hydrogenated is completely absent.

Furthermore, with neon as a carrier gas, a broad structure of low intensity is observed on the blue side of the strong and sharp peaks. Similar observations have been made before. ${ }^{26,27}$ For $\left(\mathrm{C}_{6} \mathrm{H}_{6}\right)\left(\mathrm{C}_{6} \mathrm{D}_{6}\right)$ such a broad background is not only observed to the right of the sharp peak, but also near the position where the second sharp peak is observed when using helium as carrier gas.

Although not fundamental for the discussion later in this publication, some comments should be made on those broad and weak transitions. The relative intensity of this structure is sensitive to experimental conditions. In general, a broad structure can result when the excitation occurs from and/or to several energy levels. In this specific case, hot bands as well as unfavorable Franck-Condon factors could cause such broad structure. Hot bands could explain the dependence of the observed structure on the expansion gas, as helium usually produces colder beams. Excitation of "top" molecules is known to cause a long VdW progression and to occur only a few wavenumbers blue to the transition of the "stem" excitation of the corresponding isotopomer. ${ }^{26}$ While hot band transitions usually occur red-shifted from the origin transitions, situations are conceivable where they also occur blueshifted. The observed broad structures could thus be the result of a combination of (blue-shifted) hot band transitions and "stem" excitation. In the case of the two homodimers these 
(a)

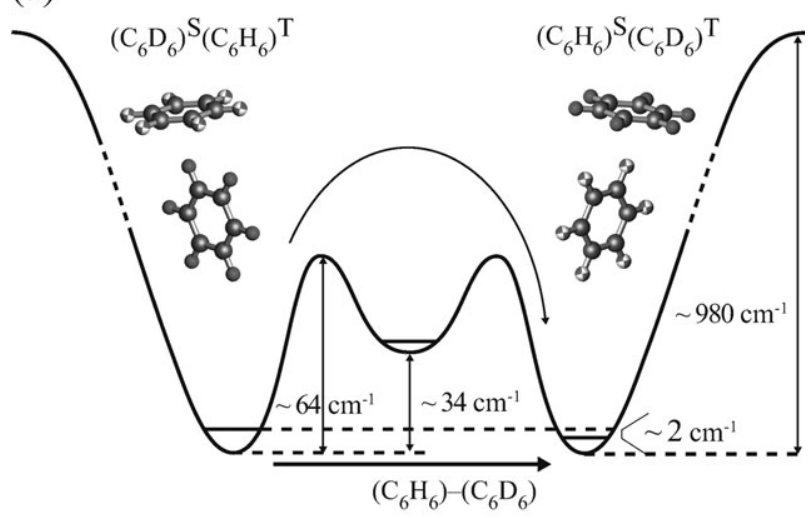

(b)

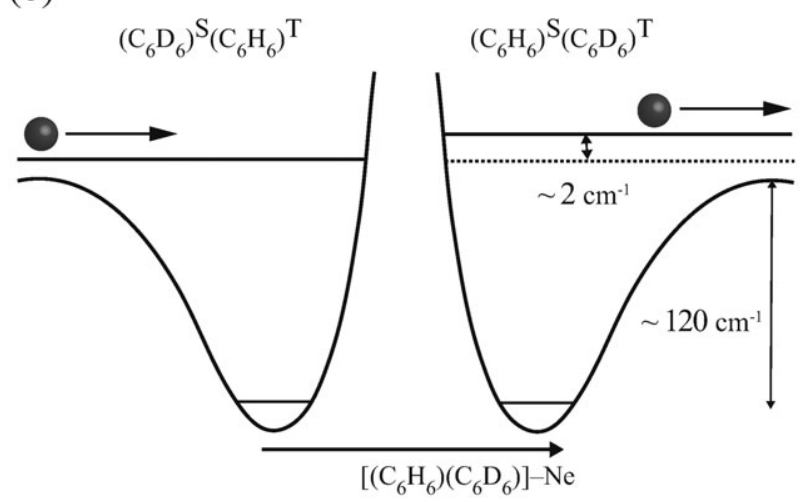

Fig. 2 (a) Scheme of the potential energy surface of the isotopically mixed benzene dimer. The two $\mathrm{T}$-shaped dimer isomers are separated by two transition states and a minimum, corresponding to a parallel displaced configuration. (b) Pictorial representation of the formation and dissociation of the neon-benzene dimer complex, converting the high energy isomer into the low energy isomer. Note that in (b), the reaction coordinate is (schematically) the distance between the benzene dimer and the neon atom.

excitations might overlap. For the mixed benzene dimer $\left(\mathrm{C}_{6} \mathrm{H}_{6}\right)^{\mathrm{S}}\left(\mathrm{C}_{6} \mathrm{H}_{6}\right)^{\mathrm{T}}$ the hot bands are observed slightly to the blue of the main peak at $38045 \mathrm{~cm}^{-1}$. The spectral features corresponding to the excitation of the "top", however, are shifted about $200 \mathrm{~cm}^{-1}$ to the blue, due to the isotope shift. It is presently not clear why this structure is apparently absent when using helium as carrier gas.

In order to explain the differences in the dominant sharp spectral features observed for $\left(\mathrm{C}_{6} \mathrm{H}_{6}\right)\left(\mathrm{C}_{6} \mathrm{D}_{6}\right)$ in helium and neon, it is instructive to consider the benzene dimer in somewhat more detail. Theory suggests a binding energy of about $980 \mathrm{~cm}^{-1} .{ }^{19}$ The barrier for the exchange of the two monomeric units can be estimated as being the barrier separating the T-shaped structure from the parallel displaced sandwich structure which is calculated to be $64 \mathrm{~cm}^{-1} 19$ (see Fig. 2(a)). When the two monomeric units are identical, their exchange will not cause a change in energy. The situation is different when the two units differ in isotopic composition. In that case, the electronic energy stays the same, however, the zero-point energy will depend on which of the monomers is in the "stem" or in the "top" position. In the "stem" molecule, hydrogen bonding causes the $\mathrm{C}-\mathrm{H}$ (or $\mathrm{C}-\mathrm{D}$ ) vibrations to shift to the red, compared to either the "top" benzene ring or to the free benzene molecule. ${ }^{29}$ Estimates based on experimental or calculated vibrational frequencies predict the zero-point energy of the $\left(\mathrm{C}_{6} \mathrm{H}_{6}\right)^{\mathrm{S}}\left(\mathrm{C}_{6} \mathrm{D}_{6}\right)^{\mathrm{T}}$ conformer to be about $2 \mathrm{~cm}^{-1}$ lower than that of the $\left(\mathrm{C}_{6} \mathrm{D}_{6}\right)^{\mathrm{S}}\left(\mathrm{C}_{6} \mathrm{H}_{6}\right)^{\mathrm{T}}$ isomer.

When the benzene molecules expand into vacuum, the adiabatic expansion causes a rapid cooling. Dimerization occurs, however, the incipient benzene dimer molecule is initially warm and cools via collisions with the buffer gas. As long as the internal energy of the dimer is above the isomerization barrier, the two subunits rapidly interchange. Once the energy drops to below this barrier, isomerization stops and the conformational distribution is frozen in. At the height of the barrier, the small difference in zero-point energy of about $2 \mathrm{~cm}^{-1}$ should have a negligible effect on the relative populations and one would expect a near equal abundance of the two conformations. This is indeed observed when helium is used as an expansion gas (see Fig. 1(b)).

Why is the conformer distribution now completely different when neon is used as an expansion gas? When the benzene dimer molecules expand into vacuum, the collision rate drops very quickly. A few nozzle diameters downstream, this rate gets so low that three-body collisions and clustering become unimportant. However, two-body collisions between the dimers and carrier gas atoms still occur frequently. As the translational temperature is then already low, those collisions occur with low energy. In such a cold collision between a benzene dimer molecule and a rare gas atom, a short lived complex is formed. The internal energy of this complex is the sum of the collision energy, the internal energy of the colliding partners before the collision and the binding energy of the complex. Late in the expansion or in the cold environment of the molecular beam, the last contribution is by far the most dominating. In the absence of a third collision partner, this complex will dissociate back to the reactants very quickly (within a few pico- to nanoseconds, depending on the system) after the formation of the complex. Usually, such collisions and the formation of transient collision complexes are thus of little consequence. However, when the internal energy of the transient complex is higher than the barriers separating the conformers, isomerization can occur. This is exactly what can happen when a benzene dimer collides with a neon atom, as schematically shown in Fig. 2. As an estimate for the internal energy of the benzene dimer-rare gas complex, the binding energy $D_{0}$ of a rare gas atom to the benzene monomer molecule can be taken, which is about $48 \mathrm{~cm}^{-1} 30$ and $120 \mathrm{~cm}^{-131}$ for helium and neon, respectively. A transient complex of the benzene dimer with neon thus has enough energy for the exchange of the monomer units while a complex with helium has not (see Fig. 2(a)). If the complex is initially formed in the "high energy" conformer $\left(\mathrm{C}_{6} \mathrm{D}_{6}\right)^{\mathrm{S}}\left(\mathrm{C}_{6} \mathrm{H}_{6}\right)^{\mathrm{T}}$, complexation with neon can induce isomerization to the "low energy" conformer $\left(\mathrm{C}_{6} \mathrm{H}_{6}\right)^{\mathrm{S}}\left(\mathrm{C}_{6} \mathrm{D}_{6}\right)^{\mathrm{T}}$. The difference in zero-point energy of $\Delta E_{\mathrm{ZPE}}$ $\approx 2 \mathrm{~cm}^{-1}$ is then available as kinetic energy for dissociating the complex, strongly enhancing the dissociation rate in that channel (see Fig. 2(b)). Under the cold conditions of the molecular beam, the initial collision energy between the dimer and the rare gas atom is on the order of $E_{\mathrm{col}} \approx 1-3 \mathrm{~cm}^{-1}$. When 
the transient collision complex is formed from an initial "high energy" isomer its energy is $E_{\mathrm{col}}+\Delta E_{\mathrm{ZPE}}\left(3-5 \mathrm{~cm}^{-1}\right)$ above the exit channel to form the "low energy" isomer, however only $E_{\mathrm{col}}$ $\left(1-3 \mathrm{~cm}^{-1}\right)$ above the exit channel to dissociate back to the reactant. When the transient collision complex is formed starting with the "low energy" isomer, its energy is with $E_{\mathrm{col}}-\Delta E_{\mathrm{ZPE}}$ either below or only slightly above the exit channel for the "high energy" isomer so that the reverse "low to high energy" isomerization is strongly disfavoured. The above model is insensitive to the exact values of the energy difference $\Delta E_{\mathrm{ZPE}}$ of the isomers involved and should thus be applicable as long as this energy difference is comparable to, or bigger than, the collision energy $E_{\mathrm{col}}$. The neon atoms thus effectively act as a catalyst for the isomerization in the benzene dimer, forming selectively the low energy isomer $\left(\mathrm{C}_{6} \mathrm{H}_{6}\right)^{\mathrm{S}}\left(\mathrm{C}_{6} \mathrm{D}_{6}\right)^{\mathrm{T}}$. This mechanism, in combination with calculated energies, ${ }^{19,30}$ also predicts that the parallel displaced sandwich isomer should not survive in the molecular beam, as already helium would catalyse its destruction.

When using argon as a carrier gas, the same behaviour as for neon is observed (not shown). This is perfectly consistent with the model, since the binding energy between argon and benzene $\left(D_{0} \approx 380 \mathrm{~cm}^{-132}\right.$ ) is even higher than in the case of neon.

This mechanism is most likely of general importance. In the cold environment of a molecular beam, the translational energy available in collisions is often not high enough to overcome barriers. The formation of a (short lived) complex with a collision partner, however, increases the internal energy by the complex binding energy which allows it to surmount barriers separating different isomers. Such a mechanism works best at low temperatures. In that case, even small energy differences can be large compared to the collision energy, strongly favouring a conversion from high to low energy isomers. The final conformational distribution can then resemble a thermal distribution at the prevailing translational temperature. Recently, it has been experimentally shown that the abundance of a particular conformer of the amino-acid phenylalanine in a supersonic expansion critically depends on the carrier gas that is being used. ${ }^{33}$ It seems likely that the above described model applies here as well. Also, it is interesting to note that there presently is a large activity in the field of cold collisions. ${ }^{34}$ Here we have demonstrated a potentially very important application of cold collisions, namely the use of cold collisions in the preparation of selected conformations of gas-phase molecules.

\section{Acknowledgements}

Financial support by the Deutsche Forschungsgemeinschaft through program SFB 450 on "Analysis and control of ultrafast photoinduced reactions" is gratefully acknowledged.

\section{References}

1 Atomic and Molecular Beam Methods, ed. G. Scoles, Oxford University Press, New York, 1988 and 1992, vol. 1 and 2.
2 T. R. Rizzo, Y. D. Park and D. H. Levy, J. Chem. Phys., 1986, 85, 6945.

3 E. G. Robertson and J. P. Simons, Phys. Chem. Chem. Phys., 2001, $3,1$.

4 R. S. Ruoff, T. D. Klots, T. Emilsson and H. S. Gutowsky, J. Chem. Phys., 1990, 93(5), 3142.

5 P. D. Godfrey, R. D. Brown and F. M. Rodgers, J. Mol. Struct., 1996, 376, 65.

6 P. D. Godfrey and R. D. Brown, J. Am. Chem. Soc., 1998, 120, 10724.

7 G. M. Florio, R. A. Christie, K. D. Jordan and T. S. Zwier, J. Am. Chem. Soc., 2002, 124, 10236.

8 P. Butz, R. T. Kroemer, N. A. Macleod and J. P. Simons, Phys. Chem. Chem. Phys., 2002, 4, 3566.

9 L. C. Snoek, T. Mourik and J. P. Simons, Mol. Phys., 2003, 101(9), 1239.

10 C. Emmelmuth, V. Dyczmons and M. A. Suhm, J. Phys. Chem. A, 2006, 110, 2906.

11 B. C. Dian, A. Longarte and T. S. Zwier, Science, 2002, 296(5577), 2369.

12 B. C. Dian, J. R. Clarkson and T. S. Zwier, Science, 2004, 303(5661), 1169.

13 T. F. Miller III, D. C. Clary and A. J. H. M. Meijer, J. Chem. Phys., 2005, 122, 244323.

14 A. Bastida, J. Zúñiga, A. Requena, B. Miguel, J. A. Beswick, J. Vigué and N. Halberstadt, J. Chem. Phys., 2002, 116, 1944.

15 P. Hobza, H. L. Selzle and E. W. Schlag, J. Chem. Phys., 1990, 93(8), 5893.

16 V. Spirko, O. Engkvist, P. Soldan, H. L. Selzle, E. W. Schlag and P. Hobza, J. Chem. Phys., 1999, 111(2), 572.

17 M. O. Sinnokrot, E. F. Valeev and C. D. Sherrill, J. Am. Chem. Soc., 2002, 124, 10887

18 Y. C. Park and J. S. Lee, J. Phys. Chem. A, 2006, 110, 5091.

19 R. Podeszwa, R. Bukowski and K. Szalewicz, J. Phys. Chem. A, 2006, 110, 10345.

20 R. A. DiStasio, Jr, G. von Helden, R. P. Steele and M. HeadGordon, Chem. Phys. Lett., 2007, DOI: 10.1016/j.cplett.2007. 02.034 .

21 K. C. Janda, J. C. Hemminger, J. S. Winn, S. E. Novick, S. J. Harris and W. Klemperer, J. Chem. Phys., 1975, 63(4), 1419.

22 J. B. Hopkins, D. E. Powers and R. E. Smalley, J. Phys. Chem., 1981, 85, 3739.

23 K. H. Fung, H. L. Selzle and E. W. Schlag, J. Phys. Chem., 1983, 87, 5113.

24 K. S. Law, M. Schauer and E. R. Bernstein, J. Chem. Phys., 1984, 81(11), 4871.

25 K. O. Börnsen, H. L. Selzle and E. W. Schlag, J. Chem. Phys., 1986, 85(4), 1726.

26 B. F. Henson, G. V. Hartland, V. A. Venturo and P. M. Felker, J. Chem. Phys., 1992, 97(4), 2189.

27 W. Scherzer, O. Krätzschmar, H. L. Selzle and E. W. Schlag, Z. Naturforsch., A: Phys. Sci., 1992, 47, 1248.

28 E. Arunan and H. S. Gutowsky, J. Chem. Phys., 1993, 98, 4294.

29 U. Erlekam, M. Frankowski, G. Meijer and G. von Helden, J. Chem. Phys., 2006, 124, 171101.

30 S. Lee, J. S. Chung, P. M. Felker, J. López Cacheiro, B. Fernández, T. Bondo Pedersen and H. Koch, J. Chem. Phys., 2003, 119(24), 12956.

31 T. Brupbacher, J. Makarewicz and A. Bauder, J. Chem. Phys., 1994, 101(11), 9736, $D_{0}$ is estimated by substracting $33 \mathrm{~cm}^{-1}$ zero point vibrational energy from the given $D_{\mathrm{e}}$ of $151 \mathrm{~cm}^{-1}$.

32 P. Hobza, O. Bludský, H. L. Selzle and E. W. Schlag, J. Chem. Phys., 1992, 97(1), 335.

33 T. Ebata, T. Hashimoto, T. Ito, Y. Inokuchi, F. Altunsu, B. Brutschy and P. Tarakeshwar, Phys. Chem. Chem. Phys., 2006, 8, 4783.

34 R. V. Krems, Int. Rev. Phys. Chem., 2005, 24(1), 99, and references therein. 